ARTIGO

\title{
Transtorno do Desenvolvimento da Coordenação: desconhecido por pais e professores
}

Viviane Aparecida Pereira dos Santos ${ }^{a}$ José Luiz Lopes Vieira ${ }^{b}$ Vânia de Fátima Matias de Souza ${ }^{c}$ Luciana Ferreira ${ }^{d}$

\section{Resumo}

O estudo teve como objetivo analisar dificuldades de aprendizagem sob a perspectiva dos transtornos das habilidades motoras, a partir da percepção de pais e de professores considerando o comportamento motor, emocional, acadêmico e atividades diárias de crianças com provável Transtorno do Desenvolvimento da Coordenação (TDC). Participaram 42 pais, 32 professores e 42 pré-escolares. Como instrumentos foram utilizados o Movement Assessment Battery for Children-2 (MABC-2) e questionários de percepção dos pais e dos professores sobre o desenvolvimento da criança. Os dados foram analisados usando o teste Shapiro Wilk, Wilcoxon, "U” de Mann-Whitney e Regressão Logística Binária. Os resultados demonstraram que professores percebem melhor as dificuldades nas habilidades motoras das crianças com persistência do TDC (pTDC) ou variação do TDC (vTDC) e percebem melhor as dificuldades emocionais, acadêmicas e de atividades diárias. Conclui-se que os professores percebem melhor as dificuldades apresentadas por crianças com pTDC e a variação no diagnóstico do transtorno interfere na percepção da dificuldade motora das crianças.

Palavras-chave: Habilidade Motora. Pais. Professores.

\section{Introdução}

As relações estabelecidas no campo da produção do conhecimento acerca das dificuldades de aprendizagem em geral apresentam-se acompanhadas das ações

\footnotetext{
a Faculdade de Filosofia Ciências e Letras de Mandaguari, Mandaguari, PR, Brasil.

b Universidad Católica del Maule, Talca, Chile.

c Universidade Estadual de Maringá, Maringá, PR, Brasil.

d Universidade Estadual de Londrina, Londrina, PR, Brasil.
} 
mediadas entre os processos de aprendizagem acopladas à questão da inclusão escolar (SANTIAGO; SANTOS; MELO, 2017). Na Lei de Diretrizes e Bases da Educação Nacional (LDBEN), Lei nº. 9.394/96, em especial em seu Artigo 59:

[...] os sistemas de ensino devem assegurar aos estudantes da Educação básica, especificamente, currículos, métodos, técnicas, recursos educativos e organização específica para atender as suas necessidades; preconiza a oferta de professores especializados para o atendimento especial e professores capacitados para inclusão desses educandos na rede regular de ensino; assegurando o atendimento da Educação especial para o trabalho além de garantir os acessos igualitários aos benefícios dos programas suplementares disponíveis para o respectivo nível de ensino regular (BRASIL, 1996).

Entretanto, para que essa ação didático-pedagógica ocorra é necessária a compreensão por parte dos envolvidos acerca das potencialidades e das fragilidades apresentadas pela criança durante todo o processo educativo. De acordo com Alves e Nakano (2015), é importante que os envolvidos conheçam e saibam identificar as dificuldades, os distúrbios e os transtornos de aprendizagem em seus alunos, em si mesmos, nas aulas e nos ambientes educacional e social escolar, afinal, a criança é um ser social e é reflexo das relações interambientais.

As dificuldades de coordenação motora, comuns em crianças em idade pré-escolar e escolar, têm ocupado lugar de destaque nas pesquisas relacionadas ao campo educacional, sendo consideradas como um dos problemas para a criança desenvolver-se de forma integral e autônoma (ALVAREZ; COUTO, 2020; CARVALHO; COELHO; TOLOCKA, 2016).

Sabe-se que a Educação Infantil é um espaço em que a ludicidade, a criatividade e o construir o conhecimento por meio das experiências e das vivências corporais consigo e com o outro oportuniza possibilidades, ainda que na informalidade, para as discussões sobre as dificuldades de aprendizagem. Destaca-se ainda que nos últimos cinco anos, o número de matrículas na Educação Infantil aumentou 12,6\%. Atualmente, mais de 8,9 milhões de crianças com até 6 anos no Brasil frequentam centros de Educação Infantil (BRASIL, 2019). A American Psychiatric Association - APA (2013) revela que 5 a $6 \%$ das crianças em idade escolar nos estados Unidos da América apresentaram Transtorno do Desenvolvimento da Coordenação (TDC) e outros $15 \%$ estão em risco de desenvolver tal transtorno. Existe no Brasil mais de 1 milhão de crianças vulneráveis a alguma desordem durante seu desenvolvimento motor. Nesse sentido, diagnosticar o TDC é uma tarefa primordial para pais, professores e profissionais da saúde nos anos iniciais (CAÇOLA; LAGE, 2019). 
O TDC que, ao contrário da crença, não é uma condição passageira da infância, pode persistir na adolescência e na vida adulta, com graves sequelas a longo prazo relacionadas às dificuldades sociais, emocionais, acadêmicas, psiquiátricas e resultados profissionais adversos (YU; BURNETT; SIT, 2018). O TDC é caracterizado por prejuízo acentuado no desenvolvimento da coordenação motora, não relacionado a problemas neurológicos, e prejudicam, efetivamente, as atividades da vida diária dos escolares, (ALESI; PECORARO; PEPI, 2018; APA, 2013).

Missiuna, Rivard e Pollock (2011) argumentam que os primeiros a notarem o problema motor são os pais e/ou professores, pois as dificuldades interferem no desempenho acadêmico e nas atividades diárias. As crianças podem apresentar dificuldades em planejar, organizar ou modificar os movimentos, dependendo de feedback, pois não aprendem com os próprios erros, além de apresentarem dificuldades em transferir uma habilidade motora para outras situações e problemas para aquisição de novas habilidades (CAÇOLA; LAGE, 2019).

Embora pais e/ou professores observem o problema motor, a falta de conhecimento dificulta a identificação do transtorno tanto em casa (MILANDER; COETZEE; VENTER, 2015) quanto na escola (MISSIUNA et al., 2006a). Outra preocupação importante a respeito das crianças com TDC é que, na maioria das vezes, os elas não são diagnosticadas formalmente, mas, sim, são descritos por seus pais e professores como "preguiçosos" ou "desajeitados" (LODH; COGHILL; FOSTER, 2017, KWAN et al., 2013; ZWICKER et al., 2012).

Apesar de alguns países terem estendido a discussão acerca do TDC (MISSIUNA et al., 2007), no Brasil, o transtorno e suas características continuam sendo fenômenos pouco explorados (BIM, VIEIRA, 2020; CAÇOLA; LAGE, 2019; LACERDA; MAGALHÃES; REZENDE, 2007). Em que pese a alta prevalência mundial, com pesquisas internacionais apontando para índices de até 22\% (CERMAK; GUBBAY; LARKIN, 2002) e, no Brasil, com valores entre 4,4\% (SOUZA et al., 2007) e 30\% (BARBA et al., 2017) em crianças com idade entre 4 e 14 anos, observa-se que o significado do TDC nos ambientes escolar e familiar é pouco conhecido e, consequentemente, visto com menor importância quando comparado a outros transtornos do desenvolvimento (FERREIRA et al., 2006).

Na literatura nacional, apenas uma pesquisa objetivou investigar a percepção sobre mães brasileiras em relação ao desempenho nas atividades diárias de crianças com TDC (GALVÃO et al., 2014a), de modo geral, todas as mães percebiam alguma dificuldade, mas não tinham conhecimento do transtorno. O mesmo acontece com o sistema escolar, onde o diagnóstico ainda é pouco conhecido (GALVÃO et al., 
2014a; PULZI; RODRIGUES, 2015), como verificado no estudo de Magalhães et al. (2009) inferindo falta de conhecimento em relação ao transtorno motor.

Com base nos argumentos apresentados, observa-se a relevância de estudos com crianças com TDC em idade pré-escolar que considerem a percepção de pais e professores visando melhorar as condições de acesso a informações, formas de intervenção e de programas que atividades motoras para a melhor qualidade de vida das crianças. Diante do exposto, pontua-se uma lacuna que busca avançar no sentido de analisar a percepção de pais e de professores acerca do comportamento motor, emocional, acadêmico e das atividades diárias de crianças com TDC.

\section{Métodos}

Abaixo o número de participantes do estudo, os instrumentos de medida e os procedimentos utilizados.

\subsection{Participantes}

Participaram do estudo 42 pais/responsáveis, 27 professores e cinco professores de Educação Física de 42 pré-escolares com idade inicial média de 3,4 $\pm 0,5$ anos, com provável TDC. A partir da avaliação motora das 773 crianças, 77 foram identificadas com suspeita de TDC. Dessas 77 crianças, 42 atendiam aos critérios de inclusão. A partir da segunda avaliação (12 meses após a primeira avaliação), foram criados dois grupos: um no qual o TDC permaneceu após 12 meses, denominado de "persistência do TDC" (pTDC), constituído por 24 crianças, e um no qual o TDC variou após 12 meses, denominado de "variação do TDC" (vTDC), constituído por 18 crianças.

Os professores e pais/responsáveis das 42 crianças identificadas com suspeita de TDC, em pelo menos um dos momentos de avaliação, foram contatados pela coordenação de cada uma das quatro creches, solicitando preenchimento dos questionários da pesquisa.

O critério de inclusão foi a criança ter duas avaliações motoras (num intervalo de 12 meses entre a primeira e segunda avaliação) que apresentassem suspeita de TDC em pelo menos uma dessas avaliações. Foram critérios de exclusão: a) o preenchimento incorreto dos questionários pelos pais/responsáveis e professores e b) crianças que apresentassem laudos com diagnósticos de transtornos ou deficiências.

\subsection{Instrumentos de medida}

$\mathrm{Na}$ avaliação do desenvolvimento motor utilizou-se a Bateria I ( 3 a 6 anos) do teste Movement Assessment Battery for Children - 2 (MABC-2) (HENDERSON; 
SUDGEN; BARNETT, 2007) ${ }^{1}$. Cada tarefa resulta em um escore padrão que é somado gerando a pontuação total do teste e origina graus de percentis para identificar o nível do desenvolvimento motor de acordo com o grau de dificuldade motora. Partindo dos critérios do teste considerou-se que crianças com pontuação $\leq 5 \%$ apresentavam problemas de coordenação motora, denominadas de provável TDC; percentil de 6 a 15 , como casos suspeitos e crianças com percentil $>15$ foram consideradas com desenvolvimento típico (DT).

Para avaliar a percepção de pais ou responsáveis sobre as habilidades motoras, acadêmicas, emocionais e comportamentais e das atividades diárias de crianças com provável TDC, utilizou-se um questionário estruturado para pais de crianças com idade entre três e cinco anos com indicativo de TDC com base nos questionários Affordances in the Home Environment for Motor Development (AHEMD), de Rodrigues, Saraiva e Gabbard (2005), que avalia a quantidade e a qualidade do ambiente familiar, e Development Coordination Disorder Questionnaire (DCDQ), de Wilson et al. (2000)². Além de informações referentes ao livreto "Crianças com Transtorno do Desenvolvimento da Coordenação: em Casa e na Sala de Aula" de Missiuna (2003), criado para auxiliar pais e professores a identificarem e lidarem com crianças que demonstrem problemas de movimento característicos do TDC.

Com base nessas referências, o instrumento visa caracterizar a população estudada com informações sociodemográficas da família, perfil biológico da criança e dados sobre a creche. Além desses elementos, o questionário possui 57 questões dicotômicas ("sim" e "não"), organizadas em quatro dimensões: habilidades motoras (21 questões), habilidades acadêmicas/escolares (sete questões), habilidades emocionais e comportamentais (19 questões) e habilidades das atividades diárias (10 questões). A pontuação dá-se pela soma das questões de cada dimensão, sendo que a resposta "não" vale "zero" e a resposta "sim" vale "um". Quanto maior a somatória de cada dimensão, e para o total das dimensões, maiores serão as dificuldades apresentadas pelas crianças.

Esse instrumento foi avaliado por especialistas em desenvolvimento motor e revelaram coeficientes de validade de conteúdo acima de 0,80 para clareza de linguagem e pertinência prática, já as dimensões avaliadas apresentaram concordância acima de 70\% e um Kappa médio de 0,92, sugerindo excelente concordância entre avaliadores (LANDIS; KOCH, 1977). Quanto à qualidade

\footnotetext{
O Movement Assessment Battery for Children validado por Valentini, Ramalho e Oliveira (2014) com as seguintes propriedades psicométricas: $\alpha=0,78$ para o teste geral, $\alpha=0,77$ para a destreza manual, $\alpha=0,52$ para a habilidade de lançar e receber, e $\alpha=0,77$ para a habilidade de equilíbrio.

2 O Questionário que avalia a suspeita de TDC foi traduzido e adaptado para Português por Prado, Magalhães e Wilson (2009).
} 
psicométrica, o questionário apresentou $\alpha=0,88$ para o teste geral, $\alpha=0,76$ na subescala de habilidade acadêmica, $\alpha=0,61$ na subescala de atividades diárias, $\alpha=0,77$ na subescala de habilidade motora e $\alpha=78$ na subescala de habilidade emocional e comportamental.

Em se tratando das percepções dos professores, as avaliações foram realizadas a partir de um questionário estruturado sobre as habilidades motoras, acadêmicas, emocionais e comportamentais e das atividades diárias de crianças com provável TDC, com idade entre três e cinco anos. Esse instrumento foi adaptado com base nos questionários: Early Years Movement Skills Checklist, de Chambers e Sugden (2006), traduzido e adaptado para o Português por Iwamizu (2013), que avalia a suspeita de TDC em crianças; o Development Coordination Disorder Questionnaire (DCDQ), de Wilson et al. (2000), traduzido e adaptado para o nosso idioma por Prado, Magalhães e Wilson (2009).

Esse instrumento coleta informações referentes à criança: data de nascimento, idade, sexo e diagnóstico médico - como algum distúrbio ou doença e aos professores: formação e escolaridade da (s) professora (s), se professora de sala de aula ou de Educação Física e número de alunos na sala de aula. Além desses dados, o questionário tem 55 questões dicotômicas ("sim" e "não"), organizadas em quatro dimensões: habilidades motoras (20 questões), habilidades acadêmicas/ escolares (sete questões), habilidades emocionais e comportamentais (19 questões) e habilidades das atividades diárias (nove questões). A pontuação dá-se pela soma das questões de cada dimensão, sendo que a resposta "não" vale "zero" e a resposta "sim" vale "um". Quanto maior a somatória de cada dimensão, e para o total das dimensões, maiores serão as dificuldades apresentadas pelas crianças.

O questionário foi avaliado por especialistas em desenvolvimento motor e revelaram coeficientes de validade de conteúdo acima de 0,80 para clareza de linguagem e pertinência prática, já as dimensões avaliadas apresentaram concordância acima de 70\% e um Kappa médio de 0,92, indicando excelente concordância entre avaliadores (LANDIS; KOCH, 1977). Quanto à qualidade psicométrica, utilizou-se o método estatístico Coeficiente Kappa médio (HERNÁNDEZ-NIETO, 2002), que avaliou a validade de conteúdo quanto à clareza de linguagem e à pertinência prática. Os resultados referentes ao Coeficiente Kappa médio foram analisados pelos pontos de corte de Landis e de Koch (1977), tendo o questionário apresentado $\alpha=0,96$ para o teste geral, $\alpha=0,87$ na subescala de habilidade acadêmica, $\alpha=0,70$ na subescala de atividades diárias, $\alpha=0,94$ na subescala de habilidade motora e $\alpha=89$ na subescala de habilidade emocional e comportamental. 


\subsection{Procedimentos}

Esse estudo foi aprovado pelo Comitê Permanente de Ética em Pesquisa com seres humanos ( $\mathrm{n}^{\circ}$ 0127.0.093.000-11), autorizado pela Secretaria de Educação e pais e professores preencheram o Termo de Consentimento Livre e Esclarecido (TCLE). A avaliação das crianças foi individual, com duração média de 20 minutos, dando oportunidade para que se familiarizassem com cada tarefa, conforme protocolo estabelecido. Os pais foram questionados via telefone e os professores, pessoalmente, acerca do conhecimento sobre o TDC.

\subsection{Análise dos dados}

Inicialmente, a partir do teste Shapiro-Wilk, verificou-se que os dados não apresentaram distribuição normal. Assim, foram utilizadas mediana (Md), quartis (Q1; Q3) e mínimo e máximo (Min-Max) para a caracterização dos resultados. Na comparação entre a percepção de pais e de professores, foi utilizado o teste Wilcoxon e para a comparação da percepção de pais e de professores entre as crianças com pTDC e vTDC foi utilizado o teste "U", de Mann-Whitney.

A Regressão Logística Binária (análise bruta e ajustada) foi usada para examinar as associações das variáveis independentes (percepção de pais, de professores em cada dimensão e para o total das dimensões) com a dependente (pTDC durante 12 meses) das crianças. Todas as variáveis foram inclusas no modelo de regressão ajustado, independentemente do valor de $p$ da análise bruta. $\mathrm{O}$ ajuste do modelo foi verificado usando o teste de Hosmer-Lemeshow $(\mathrm{p}<0,05)$.

\section{Resultados}

Sobre o conhecimento do TDC, pais e professores foram questionados e todos revelaram desconhecimento acerca do transtorno motor. A Tabela 1 apresenta a comparação entre a percepção de pais e de professores quanto à dificuldade nas habilidades acadêmicas, motoras, emocionais e nas atividades diárias de crianças com pTDC e vTDC. Para as crianças com pTDC, houve diferença, estatisticamente significativa apenas, para a percepção de dificuldades nas habilidades motoras $(\mathrm{p}=0,047)$, evidenciando maior percepção de dificuldades por professores quando comparados aos pais. Para as demais habilidades, notou-se que pais e professores apresentam percepções semelhantes. Já para as crianças com vTDC, observou-se diferença significativa nas habilidades motoras $(p=0,041)$ e emocionais $(p=0,010)$. Esses resultados apontam que os pais percebem mais dificuldades apresentadas por crianças com vTDC quando comparados aos professores. 
Tabela 1 - Comparação das percepções de pais e de professores acerca das habilidades acadêmicas, motoras, emocionais e das atividades diárias de crianças com pTDC e vTDC

\begin{tabular}{|c|c|c|c|c|c|}
\hline \multirow{3}{*}{ DIFICULDADES } & \multicolumn{4}{|c|}{ pTDC } & \multirow{3}{*}{$\boldsymbol{P}$} \\
\hline & \multicolumn{2}{|c|}{ Pais } & \multicolumn{2}{|c|}{ Professores } & \\
\hline & Md (Q1;Q3) & Min-Max & Md (Q1;Q3) & Min-Max & \\
\hline Acadêmicas & $3,0(1,0 ; 4,75)$ & $0-7$ & $4,0(1,0 ; 5,00)$ & $0-7$ & 0,244 \\
\hline Atividades diárias & $3,0(2,0 ; 5,00)$ & $0-9$ & $3,0(2,0 ; 5,00)$ & $0-7$ & 0,684 \\
\hline Motoras & $3,0(2,0 ; 5,75)$ & $0-14$ & $6,5(1,5 ; 14,00)$ & $0-18$ & $0,047^{*}$ \\
\hline \multirow[t]{2}{*}{ Emocionais } & $4,0(3,0 ; 5,00)$ & $0-13$ & $3,5(0,0 ; 8,75)$ & $0-16$ & 0,688 \\
\hline & \multicolumn{4}{|c|}{ vTDC } & \\
\hline Acadêmicas & $1,0(0,0 ; 4,00)$ & $0-6$ & $1,5(0,0 ; 5,25)$ & $0-7$ & 0,416 \\
\hline Atividades diárias & $3,0(1,0 ; 5,00)$ & $1-7$ & $2,0(1,0 ; 3,00)$ & $0-8$ & 0,051 \\
\hline Motoras & $4,0(2,0 ; 6,50)$ & $0-13$ & $1,0(0,0 ; 3,25)$ & $0-9$ & 0,041 * \\
\hline Emocionais & $4,5(3,0 ; 6,25)$ & $1-14$ & $2,0(0,0 ; 4,25)$ & $0-9$ & $0,010^{*}$ \\
\hline
\end{tabular}

*Diferença Significativa $(p<0,05)$ - Teste Wilcoxon; pTDC: Persistência do TDC; vTDC: Variação do TDC Fonte: Elaborada pelos autores (2020)

A Tabela 2 compara a percepção de pais e de professores acerca da prevalência pTDC ou vTDC nas crianças, nas habilidades acadêmicas, motoras, emocionais e das atividades diárias. Para pais, não foi identificada diferença estatisticamente, significativa nessas dimensões em função da pTDC ou vTDC nas crianças $(p>0,05)$. Já na percepção dos professores, verificou-se diferença, estatisticamente, significativa para as dificuldades nas habilidades motoras $(\mathrm{p}=0,002)$, demonstrando que os professores percebem melhor as dificuldades nas habilidades motoras nas crianças em que o TDC permaneceu (pTDC), quando comparados às crianças em que o TDC apresentou variação (vTDC).

Tabela 2 - Comparação da percepção de pais e de professores acerca das dificuldades nas habilidades acadêmicas, motoras, emocionais e das atividades diárias entre crianças com PTDC e vTDC

\begin{tabular}{|c|c|c|c|c|c|}
\hline \multirow{3}{*}{ Dificuldades } & \multicolumn{4}{|c|}{ Pais } & \multirow{3}{*}{$\boldsymbol{P}$} \\
\hline & \multicolumn{2}{|c|}{ pTDC } & \multicolumn{2}{|c|}{ vTDC } & \\
\hline & Md (Q1;Q3) & Min-Max & Md (Q1;Q3) & Min-Max & \\
\hline Acadêmicas & $3,0(1,0 ; 4,75)$ & $0-7$ & $1,0(0,0 ; 4,00)$ & $0-6$ & 0,114 \\
\hline Atividades diárias & $3,0(2,0 ; 5,00)$ & $0-9$ & $3,0(1,0 ; 5,00)$ & $1-7$ & 0,948 \\
\hline Motoras & $3,0(2,0 ; 5,75)$ & $0-14$ & $4,0(2,0 ; 6,50)$ & $0-13$ & 0,435 \\
\hline \multirow[t]{2}{*}{ Emocionais } & $4,0(3,0 ; 5,00)$ & $0-13$ & $4,5(3,0 ; 6,25)$ & $1-14$ & 0,311 \\
\hline & \multicolumn{4}{|c|}{ Professores } & \\
\hline Acadêmicas & $4,0(1,0 ; 5,00)$ & $0-7$ & $1,5(0,0 ; 5,25)$ & $0-7$ & 0,138 \\
\hline Atividades diárias & $3,0(2,0 ; 5,00)$ & $0-7$ & $2,0(1,0 ; 3,00)$ & $0-8$ & 0,063 \\
\hline Motoras & $6,5(1,5 ; 14,00)$ & $0-18$ & $1,0(0,0 ; 3,25)$ & $0-9$ & $0,002 *$ \\
\hline Emocionais & $3,5(0,0 ; 8,75)$ & $0-16$ & $2,0(0,0 ; 4,25)$ & $0-9$ & 0,184 \\
\hline
\end{tabular}

*Diferença Significativa $(p<0,05)$ - Teste "U" de Mann-Whitney; pTDC: Persistência do TDC; VTDC: Variação do TDC

Fonte: Elaborada pelos autores (2020) 
A Tabela 3 associa a percepção de pais e de professores quanto às dificuldades nas habilidades acadêmicas, motoras, emocionais e das atividades diárias e os resultados identificados no desempenho motor das crianças, utilizando como referência as crianças que persistiram no pTDC. Na análise bruta, ocorreu associação significativa apenas para a percepção dos professores quanto às dificuldades nas habilidades motoras $(\mathrm{p}=0,009)$, demonstrando que professores percebem melhor as dificuldades na coordenação motora das crianças com pTDC comparadas às crianças com vTDC. Quando a análise foi ajustada para todas as variáveis, verificou-se associação entre a pTDC com a percepção dos professores quanto às dificuldades na mesma habilidade, ou seja, motoras $(\mathrm{p}=0,012)$, demonstrando a confirmação da associação entre essas variáveis.

Para a percepção dos pais, após o ajuste do modelo, foi identificada associação da pTDC com as dificuldades nas habilidades acadêmicas $(p=0,037)$, sugerindo que as variáveis moderadoras interferiram na análise demonstrando que os pais percebem melhor as dificuldades acadêmicas em crianças com pTDC comparadas às crianças com vTDC. Esses resultados sugerem que pais e professores conseguem notar com clareza as dificuldades vivenciadas pelas crianças com pTDC.

Tabela 3 - Regressão logística binária da percepção de pais e de professores quanto às dificuldades nas habilidades acadêmicas, motoras, emocionais e das atividades diárias de crianças com pTDC

\begin{tabular}{|c|c|c|c|c|}
\hline \multirow{2}{*}{ Dificuldades } & \multicolumn{3}{|c|}{ Professores } & \multirow[b]{2}{*}{$\boldsymbol{P}$} \\
\hline & $\mathrm{OR}_{\text {bruta }}[\mathrm{IC} 95 \%]$ & $\boldsymbol{P}$ & OR $_{\text {ajustada }}$ [IC 95\%] & \\
\hline Acadêmicas & $1,190[0,928-1,525]$ & 0,171 & $0,867[0,562-1,336]$ & 0,518 \\
\hline Atividades diárias & $1,337[0,944-1,895]$ & 0,102 & $1,256[0,762-2,070]$ & 0,371 \\
\hline Motoras & $1,294[1,067-1,569]$ & $0,009 *$ & $1,099[1,077-1,818]$ & $0,012 *$ \\
\hline Emocionais & $1,176[0,982-1,409]$ & 0,077 & $0,894[0,660-1,212]$ & 0,470 \\
\hline \multirow{2}{*}{ Dificuldades } & \multicolumn{3}{|c|}{ Pais } & \\
\hline & $\mathrm{OR}_{\text {bruta }}[\mathrm{IC} 95 \%]$ & $\boldsymbol{P}$ & $\mathrm{OR}_{\text {ajustada }}$ [IC 95\%] & $\boldsymbol{P}$ \\
\hline Acadêmicas & $1,261[0,912-1,745]$ & 0,161 & $1,559[1,027-2,366]$ & $0,037^{*}$ \\
\hline Atividades diárias & $1,022[0,748-1,396]$ & 0,892 & $1,249[0,808-1,931]$ & 0,317 \\
\hline Motoras & $0,936[0,780-1,124]$ & 0,479 & $0,877[0,668-1,151]$ & 0,344 \\
\hline Emocionais & $0,935[0,782-1,118]$ & 0,464 & $0,824[0,637-1,067]$ & 0,142 \\
\hline
\end{tabular}

*Associação significativa $-p<0,05$ : Regressão Logística Binária. OR ajustada por todas as variáveis da análise bruta independente do valor de $\mathrm{p}$.

OR: Odds Ratio; IC: Intervalo de Confiança

Fonte: Elaborada pelos autores (2020) 


\section{Discussão}

Os resultados do presente estudo apontaram que $100 \%$ dos pais e dos professores não conhecem o TDC. Tal desconhecimento pode gerar má interpretação das dificuldades apresentadas pela criança, fazendo-os acreditar que possam ser culpadas pelas características desajeitadas que apresenta (ROSENBLUM; ENGEL-YEGER, 2014). No âmbito escolar, as reações dos professores à discrepância entre o potencial acadêmico e o desempenho motor, o comportamento, a falta de atenção ou a evasão de tarefas podem ser semelhantes às reações de pais que ainda não compreenderam a natureza da condição de seu filho (MISSIUNA et al., 2006a).

Essa falta de compreensão acerca das razões pelas quais uma criança apresenta problemas com tarefas especificas pode rotulá-las como "preguiçosas" ou "não-cooperativas" (LODH; COGHILL; FOSTER, 2017; KWAN et al., 2013), levando ao agravamento do problema motor. Essas características podem originar problemas de ordem secundária nos portadores de TDC, tais como diminuição da autoestima, ansiedade, depressão e também isolamento social, interferindo consideravelmente em sua qualidade de vida (DRAGHI et al., 2020; ZWICKER et al., 2012). Dessa forma, pelo fato do TDC não ser uma condição reconhecida, o mal entendimento pode ser perpetuado (MISSIUNA et al., 2006a).

A partir dessa informação, os resultados apontaram que, apesar do desconhecimento sobre o TDC, os professores, quando comparados aos pais, perceberam melhor as dificuldades nas habilidades motoras apresentadas por crianças com pTDC, enquanto os pais perceberam mais dificuldades nas habilidades motoras e emocionais em crianças com vTDC (Tabela 1). Ainda, ao comparar as dificuldades de desenvolvimento entre crianças com persistência (pTDC) e variação do TDC (vTDC) na percepção de pais e de professores, foram observadas diferenças significativas nas habilidades motoras, somente na perspectiva dos professores, evidenciando mais dificuldades apresentadas pelas crianças com pTDC (Tabela 2). O difícil reconhecimento das dificuldades motoras por parte dos pais pode ser decorrente do fato de que essas dificuldades se apresentam de forma mais sutil nesse período da vida. No entanto, com o ingresso no sistema escolar, os problemas motores se tornam mais perceptíveis, aumentando as chances de reconhecimento do TDC (HARROWELL et al., 2018). Esses aspectos foram evidenciados em pesquisas (JARANI; NICAJ, 2014; MISSIUNA et al., 2006a, 2006b, 2007), nas quais observou-se a percepção dos pais quanto às experiências iniciais com crianças com TDC, evidenciando as dificuldades dos pais em identificar os problemas motores em idade pré-escolar. 
Ademais, as alterações sociais, demográficas e econômicas que ocorreram no século XX, tendo inserido a mulher no mercado de trabalho, diminuíram a disponibilidade dos pais aos filhos (COSTA, 2018; SARAIVA; WAGNER, 2013; VERÍSSIMO et al., 2015). Segundo Genadek e Hill (2017) a maioria dos pais possui agendas sobrecarregadas e pouco tempo disponível para engajar-se em atividades com seus filhos em situações cotidianas (como almoçar, jantar, tomar o café da manhã, ler uma história ao ir para a cama etc.). Essas práticas com crianças menores ocorrem nas creches de tempo integral, e quando as crianças estão em casa, na maioria das vezes, os pais estão atarefados com os serviços domésticos e relacionados ao trabalho.

Keizer et al. (2020) reporta que o trabalho dos pais requer dispêndio de grande quantidade de tempo e de energia fora do lar, implicando menor participação direta na vida familiar e, com isso, podem levar à menor percepção de problemas de coordenação motora, uma vez que o TDC não apresenta uma condição clínica (APA, 2013) além de se apresentar de modo ameno na primeira infância (MISSIUNA et al., 2007). Tais aspectos podem justificar o menor reconhecimento dos problemas motores pelos pais, quando comparados aos professores, os quais têm maior envolvimento nas atividades cotidianas das crianças menores.

Além da desgastante jornada de trabalho dos pais, muitos deles não vivem em união estável, o que pode diminuir o contato das crianças com o pai (OLIVEIRA; CREPALDI, 2018). A ausência do pai no lar indica também a rara cooperação nos cuidados infantis, ocasionada pela restrita comunicação estabelecida pelo antigo casal (GENADEK; HILL, 2017), o que, consequentemente, sobrecarregará a mãe, que, além de trabalhar fora, é responsável, na maioria das vezes, pelos cuidados com a criança e com os afazeres domésticos, ocasionando uma menor percepção materna em relação aos problemas associados ao desenvolvimento da criança. Contudo, muitos pais, mesmo que presentes no lar, não se envolvem diretamente com os cuidados, principalmente corporais e brincadeiras, mostrando-se ausentes afetivamente (KEIZER et al., 2020), contribuindo para que os problemas de coordenação motora da criança passem despercebidos.

Em contrapartida, evidências nos estudos demonstram que professores fazem julgamentos mais precisos e confiáveis sobre as habilidades das crianças (JUNAID et al., 2000), como revelado por Cueto et al. (2017) e Gehris, Goose and Whitaker (2014) ao examinarem as habilidades de professores para identificação de déficits motores nos escolares. Os achados apontaram evidências de que a maioria dos professores foram precisos para reconhecer a deficiência motora em seus alunos, além dessa informação estar correlacionada com as observações de 
pediatras e de psicólogos que utilizaram métodos de avaliação mais estruturados. Todavia, Cueto et al. (2017) ressaltam que 13,27\% dos professores avaliaram algumas crianças com desenvolvimento típico, mas elas apresentaram déficits de desenvolvimento, e a magnitude desse erro pode obscurecer o desenvolvimento, colocando em risco uma avaliação correta do processo de desenvolvimento da maturidade da criança e, assim, ameaçar a qualidade de vida posterior.

Essas pesquisas corroboram as características do ambiente da creche que os pré-escolares do presente estudo frequentavam, uma vez que se tratavam de instituições de período integral, nas quais o tempo de contato entre professores e alunos era cerca de 9 a 11 horas por dia. Esse tempo de envolvimento possibilita aos professores excelentes oportunidades para observar as crianças, interagindo com seus pares nas atividades recreativas/esportivas e em sala de aula, percebendo a forma como se comportam motoramente e a forma como se relacionam com os colegas nas atividades propostas, facilitando, dessa forma, a identificação de problemas de motricidade fina ou tarefas motoras grossas (CUETO et al., 2017; FAUGHT et al., 2008; MISSIUNA et al., 2006a, 2006b). No entanto, a idade dos alunos pode causar confusão nas interpretações dos professores: nos primeiros anos da vida de uma criança, os professores consideram que os alunos estão em constante desenvolvimento, pois observam como cada um define seu próprio ritmo, portanto, minimizam possíveis atrasos (CUETO et al., 2017)

Outra pesquisa a reforçar os resultados desse estudo foi realizada por Magalhães et al. (2009), que verificaram que $63 \%$ dos professores das escolas públicas e privadas observam problemas de coordenação motora em crianças de 4 a 8 anos. Esses resultados chamam a atenção para o fato de que professoras com diferentes formações e perspectivas teóricas sobre Educação reportam lidar rotineiramente com crianças com algum tipo de dificuldade de coordenação motora, sendo importante usar recursos que permitam a identificação dessas crianças.

É importante mencionar a participação de professores de sala de aula e de Educação Física no presente estudo, o que enriquece os resultados encontrados, ao considerar que eles são capazes de identificar e de comparar diferentes níveis de habilidades motoras (FLETCHER; MANDIGO, 2012; LARKIN; ROSE, 2005). Em pesquisa desenvolvida por Piek e Edwards (1997), ficou evidente que os professores de Educação Física (49\%) foram mais sensíveis na identificação do TDC quando comparados aos professores de sala de aula (25\%). Contudo, é importante considerar tanto as observações de professores de sala de aula quanto as dos professores de Educação Física, haja vista o professor de sala de aula vivenciar habilidades diferentes: os de sala de aula conhecem a habilidade de 
motricidade fina, enquanto os professores de Educação Física vivenciam mais as habilidades motoras grossas, além de acompanharem o comportamento e as emoções vivenciadas nessas atividades.

$\mathrm{Na}$ associação entre a percepção de pais e de professores e os resultados no desempenho motor das crianças na bateria de avaliação motora (MABC-2), os resultados dos pré-escolares com pTDC estiveram associados de forma significativa apenas para os professores nas habilidades motoras (Tabela 3). Tal associação pode ser justificada devido a professores terem oportunidade de observar as crianças em diferentes atividades motoras e por um longo período de tempo (FAUGHT et al., 2008), além de ter outras crianças como ponto de referência para comparação (CRANE; SUMNER; HILL, 2017; JUNAID et al., 2000). E o fato de as crianças comportarem-se de maneira diferente em casa e na escola pode estar relacionado à referência mais ampla e, potencialmente mais precisa, que os professores têm quando comparam essas mesmas crianças (CRANE; SUMNER; HILL, 2017).

Quanto aos pais, os resultados apontaram que a percepção e o resultado do teste motor dos pré-escolares com pTDC estiveram associados de forma significativa apenas nas habilidades acadêmicas (Tabela 3). A literatura indica que os pais são capazes de identificar os problemas que seus filhos enfrentam, contudo, apresentam dúvidas sobre como e quando abordar suas preocupações (CAÇOLA; LAGE, 2019; MEDEIROS et al., 2019; GALVÃO et al., 2014b). Nessa perspectiva, estudos (CAÇOLA, LAGE, 2019; MISSIUNA et al., 2006a) reportam que os pais mencionam a identificação das dificuldades em seus filhos, principalmente na área acadêmica (rabiscar com um lápis de cera ou brincar com um brinquedo) e nas atividades diárias de autocuidado, como se vestir ou usar talheres.

Com relação à área acadêmica, Ahern (2000) e Missiuna et al. (2006a) apontam que a escola parece funcionar como um marco, uma vez que as crianças são confrontadas com padrões mais rigorosos de desempenho e, inevitavelmente, comparadas com seus pares. Por essa razão, o diagnóstico ou a confirmação dos problemas motores acontece quando a criança se insere no contexto escolar, o que infere a necessidade de os professores compreenderem as possíveis razões para comportamentos subjacentes, tais como desatenção, ativação ou evitação de tarefas, o que parece ser a chave para a identificação das dificuldades de coordenação (DORNELAS; MAGALHÃES, 2016; MISSIUNA et al., 2006a).

Esse estudo mostra-se relevante pelo desenho longitudinal e pela inclusão de número representativo de crianças com TDC, além da investigação da 
percepção de pais e de professores sobre as dificuldades apresentadas pelas crianças. Apesar dessas contribuições, há algumas limitações: a) em relação ao diagnóstico do TDC usando apenas a bateria de testes motores; b) a ausência de instrumentos para identificação das diferentes variáveis investigadas na percepção de pais e professores e c) a falta de um grupo controle de crianças com desenvolvimento típico.

\section{Conclusão}

Esse estudo revelou que a totalidade de pais e de professores não têm conhecimento acerca do TDC. Contudo, mesmo sem o conhecimento do transtorno, os professores demonstraram maior capacidade para reconhecer as dificuldades apresentadas por crianças pré-escolares com persistência de indicativo de TDC. As dificuldades vivenciadas por crianças que apresentaram pTDC foram mais visíveis aos professores quando comparadas às crianças que apresentaram vTDC. Além disso, o estudo revelou que o problema motor resultante da avaliação, a partir da bateria motora (MABC-2), relacionou-se à percepção dos professores nas habilidades motoras, já para os pais, foi nas habilidades acadêmicas, demonstrando que professores e pais conseguem perceber que existem problemas motores nessas áreas de desenvolvimento (acadêmicas e motoras).

Esse estudo apresenta implicações práticas como a identificação precoce de crianças com TDC por especialistas para implementação de estratégias a fim de promover programas de intervenção para a casa, para a sala de aula e para o sistema educacional. Para tanto, é preciso dar apoio a pais e a professores na identificação e na compreensão do TDC a partir de palestras, de consultoria e de inclusão de projetos nas escolas, bem como, parcerias com universidades. 


\section{Coordination Development Disorder: unknown by parents and teachers}

\section{Abstract}

The study aimed to analyze learning difficulties from the perspective of motor skills disorders considering the perception of parents and teachers about the motor, emotional, academic and daily activities of children with probable Developmental Coordination Disorder (DCD). 42 parents, 32 teachers and 42 preschoolers participated. As instruments, the Movement Assessment Battery for Children (MABC-2) and questionnaires for parents and teachers about child's development were used. Data were analyzed using Shapiro Wilk, Wilkoxon, Mann-Whitney " $U$ ”' test and Binary Logistic Regression. The results showed that teachers better understand the difficulties in motor skills of children with persistent DCD $(p D C D)$ or variation on DCD ( $v D C D)$ and better understand the emotional, academic difficulties and the difficulties related to daily activities. In conclusion, teachers show a better perception of the difficulties presented by children with $p D C D$ and the variation in the diagnosis of DCD interferes in the perception of the children's motor difficulties.

Keywords: Motor skill; Parents; Teachers.

\section{Trastorno del Desarrollo de la Coordinación: desconocido por los padres y los maestros}

\section{Resumen}

El estudio tuvo como objetivo analizar las dificultades de aprendizaje desde la perspectiva de los trastornos de la motricidad, desde la percepción de padres y maestros considerando la conducta motora, emocional, académica y las actividades diarias de los niños con probable Trastorno de Coordinación del Desarrollo (TCD). Participaron 42 padres, 32 maestros y 42 niños en edad preescolar. Los instrumentos que utilizados fueron el Movement Assessment Battery for Children-2 (MABC-2) y cuestionarios sobre la percepción de padres y maestros sobre el desarrollo del niño. Los datos se analizaron mediante la prueba de Shapiro Wilk, Wilcoxon, "U” de Mann-Whitney y de Regresión Logística Binaria. Los resultados mostraron que los maestros perciben mejor las dificultades en las habilidades motoras de los niños con persistencia del TDC (pTDC) o variación del TDC (vTDC) y perciben mejor las dificultades emocionales, académicas y de actividades diarias. Se concluye que los docentes perciben mejor las dificultades que presentan los niños con pDCD y la variación en el diagnóstico del trastorno interfiere en la percepción de la dificultad motora de los niños.

Palabras clave: Habilidad Motora. Padres. Maestros. 


\section{Referências}

AHERN, K. "Something is wrong with my child": a phenomenological account of a search for a diagnosis. Early Education \& Development, Philadelphia, v. 11, n. 2, p. 187-201, 2000. https://doi.org/10.1207/s15566935eed1102_4

ALESI, M.; PECORARO, D.; PEPI, A. Executive functions in kindergarten children at risk for developmental coordination disorder. European Journal of Special Needs Education, Chichester, v. 34, n. 3, p. 285-296, 2019. https://doi.org/10.1080/08856257.2018.1468635

ÁLVAREZ, Y. A.; COUTO, J. M. P. Importancia percibida de la motricidad en Educación Infantil en los centros educativos de Vigo (España). Educação e Pesquisa, São Paulo, v. 46, e207294, 2020. https://doi.org/10.1590/S1678-4634202046207294

ALVES, R. J. R.; NAKANO, T. C. Criatividade em indivíduos com transtornos e dificuldades de aprendizagem: revisão de pesquisas. Psicologia Escolar e Educacional, São Paulo, v. 19, n. 1. p. 87-96, jan./abr. 2015. https://doi.org/10.1590/2175-3539/2015/0191802

AMERICAN PSYCHIATRIC ASSOCIATION - APA. Diagnostic and statistical manual of mental disorders. 5th. ed. Arlington, 2013.

BARBA, P. C. S. D. et al. Prevalence of Developmental Coordination Disorder signs in children 5 to 14 years in São Carlos. Motricidade, Ribeiro de Pena, v. 13, n. 3, p. 22-30, 2017. https://doi.org/10.6063/motricidade.10058

BIM, R. H.; VIEIRA, J. L. L. Desordem coordenativa desenvolvimental em crianças de Escolas Públicas de tempo parcial e integral. Journal of Human Growth and Development, [s. 1.], v. 30, n. 1, p. 104-110, jan./abr. 2020. https://doi.org/10.7322/jhgd.v30.9959

BRASIL. Lei no 9.394, de 20 de dezembro de 1996. Estabelece as diretrizes e bases da educação nacional. Diário Oficial da União, Brasília, DF, 23 dez. 1996.

BRASIL. Ministério da Educação. Matrículas na educação infantil aumentam 12,6\% nos últimos cinco anos. Brasília, DF, 2019. Disponível em: http://portal.mec.gov.br/component/tags/tag/31947-educacao-infantil. Acesso em: 8 abril. 2020. 
CAÇOLA, P.; LAGE, G. Developmental Coordination Disorder (DCD): an overview of the condition and research evidence. Motriz, Rio Claro, v. 25, n. 2, e101923, 2019. https://doi.org/10.1590/s1980-6574201900020001

CARVALHO, A. F.; COELHO, V. A. C.; TOLOCKA, R. E. Professores de educação infantil e temas sobre inclusão de crianças com deficiência no ensino regular. Educação e Pesquisa, São Paulo, v. 42, n. 3, p. 713-726, jul./set. 2016. https://doi.org/10.1590/S1517-9702201609151344

CERMAK, S.; GUBBAY, S.; LARKIN, D. What is developmental coordination disorder? Clifton Park: Delmar, 2002.

CHAMBERS, M.; SUGDEN, D. Early years movement skills: description, diagnosis and intervention. London: Whurr, 2006.

CRANE, L.; SUMNER, E.; HILL, E. L. Emotional and behavioural problems in children with Developmental Coordination Disorder: exploring parent and teacher reports. Research in Developmental Disabilities, [s. 1.], v. 70, p. 67-74, Nov. 2017. https://doi.org/10.1016/j.ridd.2017.08.001

COSTA, F. A. Mulher, trabalho e família: os impactos do trabalho na subjetividade da mulher e em suas relações familiares. Pretextos, Belo Horizonte, v. 3, n. 6, p. 434-452, set. 2018.

CUETO, S. et al. Teachers' perceptions of preschool children's psychomotor development in Spain. Perceptual and Motor Skills, Louisvlle, v. 124, n. 4, p. 1-15, Aug. 2017. https://doi.org/10.1177/0031512517705534

DRAGHI, T. T. G. et al. Sintomas de ansiedade e depressão em crianças com transtorno do desenvolvimentoda coordenação: uma revisão sistemática. Jornal de Pediatria, Rio de Janeiro, v. 96, n. 1, p. 8-19, jan/fev. 2020. https://doi.org/10.1016/j.jped.2019.03.002

DORNELAS, L. F.; MAGALHÃES, L. C. Desempenho funcional de escolares que receberam diagnóstico de atraso do desenvolvimento neuropsicomotor até os dois anos. Revista Paulista de Pediatria, São Paulo, v. 34, n. 1, p. 78-85, jan./mar. 2016. https://doi.org/10.1016/j.rppede.2015.10.001

FAUGHT, B. et al. Screening for motor coordination challenges in children using teacher ratings of physical ability and activity. Human Movement Science, Amsterdam, v. 27, p. 177-189, Apr. 2008. https://doi.org/10.1016/j.humov.2008.02.001 
FERREIRA, L. F. et al. Desordem da coordenação do desenvolvimento. Motriz, Rio Claro, v. 12, n. 3, p. 283-292, set./dez. 2006.

FLETCHER, T.; MANDIGO, J. The primary schoolteacher and physical education: a review of research and implications for Irish physical education. Irish Educational Studies, [s. 1.], v. 31, n. 3, p. 363-376, 2012. https://doi.org/10.1080/03323315.2012.710063

GALVÃO, B. A. P. et al. Percepção materna do desempenho de crianças com transtorno do desenvolvimento da coordenação. Psicologia em Estudo, Maringá, v. 19, n. 3, p. 527-538, set. 2014a. https://doi.org/10.1590/1413-73722039315

GALVÃO, B. A. P. et al. Perspectiva dos pais sobre as consequências funcionais do transtorno do desenvolvimento da coordenação (TDC): revisão da literatura. Cadernos de Terapia Ocupacional, São Carlos, v. 22, n. 1, p. 187-193, 2014b. https://doi.org/10.4322/cto.2014.020

GEHRIS, J. S.; GOOZE, R. A.; WHITAKER, R. C. Teachers' perceptions about children's movement and learning in early childhood education programmes. Child: Care, Health and Development, Oxford, v. 41, n. 1, p. 122-131, Jan. 2014. https://doi.org/10.1111/cch.12136

GENADEKA, K.; HILL, R. Parents' work schedules and time spent with children. Community Work Fam, Abingdon, v. 20, n. 5, p. 523-542, 2017. https://doi.org/10.1080/13668803.2017.1371672

HARROWELL, I. et al. The impact of developmental coordination disorder on educational achievement in secondary school. Research in Developmental Disabilities, New York, v. 72, p. 13-22, Oct. 2018. https://doi.org/10.1016/j.ridd.2017.10.014

HENDERSON, S.; SUDGEN, D.; BARNETT, A. Movement assessment battery for children: second edition (Movement ABC-2). London: Harcourt Assessment, 2007.

HERNÁNDEZ-NIETO, R. A. Contribuciones al análisis estadístico: sensibilidad (estabilidad y consistencia) de varios coeficientes de variabilidad relativa y el coeficiente de variación proporcional (CVP). el coeficiente de validez de contenido (CVC) y el coeficiente Kappa, en la determinación de la validez de contenido según la técnica de juicio de expertos. Mérida: Universidad de los Andes/IESINFO, 2002. 
IWAMIZU, J. S. Tradução, adaptação transcultural, validade e fidedignidade de um instrumento para identificação do perfil motor de crianças entre 3 e 5 anos de idade. Dissertação (Mestrado em Ciências do Movimento Humano) Universidade de São Paulo, São Paulo, 2013.

JARANI, J.; NICAJ, G. Development coordination disorder in children a parent and teacher guide for early identification (literature review). Academic Journal of Interdisciplinary Studies, [s. 1.], v. 2, n. 4, p. 203-207, July 2014. https://doi.org/10.5901/ajis.2014.v3n4p203

JUNAID, K. et al. Teachers' use of the MABC checklist to identify children with motor cordination difficuties. Pediatric Physical Therapy, Baltimore, v. 12, n. 4, p. 158-163, Winter 2000.

KEIZER, R. et al. The influence of fathers and mothers equally sharing childcare responsibilities on children's cognitive development from early childhood to school age: an overlooked mechanism in the intergenerational transmission of (dis)advantages? European Sociological Review, Oxford, v. 36, n. 1, p. 1-15, Feb. 2020. https://doi.org/10.1093/esr/jcz046

KWAN, M. et al. Understanding physical activity and motivations for children with developmental coordination disorder: an investigation using the theory of planned behavior. Research in Developmental Disabilities, New York, v. 34, n. 11, p. 3691-3698, Nov. 2013. https://doi.org/10.1016/j.ridd.2013.08.020

LACERDA, T. T. B.; MAGALHÃES, L. C.; REZENDE, M. B.

Validade de conteúdo de questionários de coordenação motora para pais e professores. Revista de Terapia Ocupacional da Universidade de São Paulo, São Paulo, v. 18, n. 2, p. 63-77, 2007. https://doi.org/10.11606/issn.2238-6149.v18i2p63-77

LANDIS, R.; KOCH, G. The measurement of observer agreement for categorical data. Biometrics, Alexandria, v. 33, n. 1, p. 159-174, Mar. 1977.

LARKIN, D.; ROSE, E. Assessment of developmental coordination disorder. In: SUGDEN, D.; CHAMBERS, M. (org.). Children with developmental coordination disorder. London: Whurr, 2005. p. 135-154.

LODH, R.; COGHILL, J.; FOSTER, H. The clumsy child: juggling diagnosis and signposting to management. Paediatrics and Child Health, Oxford, v. 27, n. 6, p. 276-280, June 2017. https://doi.org/10.1016/j.paed.2017.02.006 
MAGALHÃES, L. C. et al. Problemas de coordenação motora em crianças de 4 a 8 anos: levantamento baseado no relato de professores. Revista de Terapia Ocupacional da Universidade de São Paulo, São Paulo, v. 20, n. 1, p. 20-28, 2009. https://doi.org/10.11606/issn.2238-6149.v20i1p20-28

MEDEIROS, C. C. M. et al. Transcendendo o problema: percepções de mães e crianças sobre o impacto do transtorno do desenvolvimento da coordenação no dia a dia. Cadernos Brasileiro de Terapia Ocupacional, São Carlos, v. 27, n. 4, p. 792-805, out./dez. 2019. https://doi.org/10.4322/2526-8910.ctoAO1818

MILANDER, M.; COETZEE, F.; VENTER, A. The ability of parents to identify grade 1-learners with developmental coordination disorder at home. South African Journal for Research in Sport, Physical Education and Recreation, v. 37, n. 3, p. 55-68, 2015.

MISSIUNA, C. Crianças com transtorno do desenvolvimento da coordenação: em casa e na sala de aula. Hamilton: CanChild - Centre for Childhood Disability Research, 2003.

MISSIUNA, C. et al. A trajectory of troubles: parents' impressions of the impact of developmental coordination disorder. Physical \& Occupational Therapy in Pediatrics, New York, v. 27, n. 1, p. 81-101, 2007.

MISSIUNA, C. et al. "Missed and misunderstood": children with coordination difficulties in the school system. International Journal of Special Education, [s. 1.], v. 21, n. 1, p. 53-67, Jan. 2006b.

MISSIUNA, C. et al. Parental questions about developmental coordination disorder: a synopsis of current evidence. Paediatrics and Child Health, Oakville, v. 11 , n. 8, p. 507-512, Oct. 2006a. https://doi.org/10.1093/pch/11.8.507

MISSIUNA, C.; RIVARD, L.; POLLOCK, N. Crianças com transtorno do desenvolvimento da coordenação: em casa, na sala de aula e na comunidade. CanChild - Centre for Childhood Disability Research, n. 905, p. 1-12, 2011.

OLIVEIRA, J. L. A. P.; CREPALDI, M. A. Relação entre o pai e os filhos após o divórcio: revisão integrativa da literatura. Actualidades en Psicología, San José, v. 32, n. 124, p. 91-109, 2018. https://doi.org/10.15517/ap.v32i124.29021

PIEK, J.; EDWARDS, K. The identification of children with developmental coordination disorder by class and physical education teachers. British Journal of Educational Psychology, Edinburgh, v. 67, pt. 1, p. 55-67, Mar. 1997. https://doi.org/10.1111/j.2044-8279.1997.tb01227.x 
PRADO, M. S.; MAGALHÃES, L. C.; WILSON, B. Cross cultural adaptation of the developmental coordination questionnaire for Brazilian children. Revista Brasileira de Fisioterapia, São Carlos, v. 13, n. 3, p. 236-246, June 2009. https://doi.org/10.1590/S1413-35552009005000024

PULZI, W.; RODRIGUES, G. M. Transtorno do Desenvolvimento da Coordenação: uma revisão de literatura. Revista Brasileira de Educação Especial, Bauru, v. 21 n. 3, p. 433-444, set. 2015. https://doi.org/10.1590/S1413-65382115000300009

RODRIGUES, L. P.; SARAIVA, L.; GABBARD, C. Development and construct validation of na inventory for assessing the home environment for motor development. Research Quarterly for Exercise and Sport, [s. 1.], v. 76, n. 2, p. 140-148, July 2005. https://doi.org/10.1080/02701367.2005.10599276

ROSENBLUM, S.; ENGEL-YEGER, B. Predicting participation in children with DCD. Current Developmental Disorders Reports, Cham, v. 1, p.109-117, 2014.

SANTIAGO, M. C.; SANTOS, M. P.; MELO, S. C. Inclusão em educação: processos de avaliação em questão. Ensaio: Avaliação e Políticas Públicas em Educação, Rio de Janeiro, v.25, n. 96, p. 632-651, jul./set. 2017. https://doi.org/10.1590/S0104-40362017002500652

SARAIVA, L. A.; WAGNER, A. A Relação Família-Escola sob a ótica de professores e pais de crianças que frequentam o ensino fundamental. Ensaio: Avaliação e Políticas Públicas em Educação, Rio de Janeiro, v. 21, n. 8, p. 739-772, dez. 2013. https://doi.org/10.1590/S0104-40362013000400006

SOUZA, C. et al. O teste ABC do movimento em crianças de ambientes diferentes. Revista Portuguesa de Ciências do Desporto, Porto, v. 7, n. 1, p. 36-47, jan. 2007. https://doi.org/10.5628/rpcd.07.01.36

VALENTINI, N. C; RAMALHO, M. H.; OLIVEIRA, M. Movement assessment battery for children-2: translation, reliability, and validity for Brazilian children. Research in Developmental Disabilities, New York, v. 35, n. 3, p. 733-740, Mar. 2014. https://doi.org/10.1016/j.ridd.2013.10.028

VERÍSSIMO, M. et al. Percepções parentais acerca dos conflitos e benefícios associados com a gestão da família e do trabalho. Diaphora, Porto Alegre, v. 13, n. 1, p. 1-8, jan./jul. 2015.

WILSON, B. et al. Reliability and validity of a parent questionnaire on childhood motor skills. The American Journal of Occupational Therapy, Boston, v. 54, n. 5, p. 484-493, Sep./Oct. 2000. https://doi.org/10.5014/ajot.54.5.484 
YU, J. J.; BURNETT, A. F.; SIT, C. H. Motor skill interventions in children with developmental coordination disorder: a systematic review and meta-analysis. Archives of Physical Medicine and Rehabilitation, Philadelphia, v. 99, n. 10, p. 2076-2099, Oct. 2018. https://doi: 10.1016/j.apmr.2017.12.009

ZWICKER, J. et al. Developmental coordination disorder: a review and update. European Journal of Paediatric Neurology, London, v. 16, n. 6, p. 573-581, Nov. 2012. https://doi.org/10.1016/j.ejpn.2012.05.005

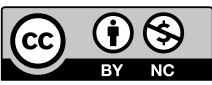

\section{Informações sobre os autores}

Viviane Aparecida Pereira dos Santos: Doutora em Educação Física pela Universidade Estadual de Maringá. Professora da Faculdade de Filosofia Ciências e Letras de Mandaguari. Contato: vivi.aps01@gmail.com

(iD) http://orcid.org/0000-0002-3979-8246

José Luiz Lopes Vieira: Doutor em Educação Física pela Universidade Federal de Santa Maria. Professor Pesquisador da Universidad Católica del Maule. Contato: jllopesvieira@gmail.com

iD https://orcid.org/0000-0003-0453-8185

Vânia de Fátima Matias de Souza: Doutora em Educação pela Universidade Estadual de Maringá. Professora Associada da mesma universidade. Contato: vfmatias@gmail.com (iD) https://orcid.org/0000-0003-4631-1245

Luciana Ferreira: Doutora em Educação Física pela Universidade Estadual de Maringá. Professora da Universidade Estadual de Londrina. Contato: luferreira@uel.com

iD http://orcid.org/0000-0001-5808-2334 